\title{
Association between edible mushroom intake and the prevalence of newly diagnosed non-alcoholic fatty liver disease: results from the Tianjin Chronic Low-Grade Systemic Inflammation and Health Cohort Study in China
}

\author{
Shunming Zhang ${ }^{1} \dagger$, Yeqing $\mathrm{Gu}^{1}{ }^{1}$, Min $\mathrm{Lu}^{2}$, Jingzhu Fu${ }^{1}$, Qing Zhang ${ }^{3}$, Li Liu ${ }^{3}$, Ge Meng ${ }^{1}$, Zhanxin $\mathrm{YaO}^{1,4}$, \\ Hongmei Wu ${ }^{1}$, Xue Bao ${ }^{1}$, Shaomei Sun ${ }^{3}$, Xing Wang ${ }^{3}$, Ming Zhou ${ }^{3}$, Qiyu Jia ${ }^{3}$, Kun Song ${ }^{3}$, Yuntang Wu ${ }^{1}$ and \\ Kaijun $\mathrm{Niu}^{1,3,5,6 *}$ \\ ${ }^{1}$ Nutritional Epidemiology Institute and School of Public Health, Tianjin Medical University, Tianjin 300070, People's Republic \\ of China \\ ${ }^{2}$ Department of Gastroenterology, Tianjin Third Central Hospital, Tianjin 300170, People's Republic of China \\ ${ }^{3}$ Health Management Centre, Tianjin Medical University General Hospital, Tianjin 300052, People's Republic of China \\ ${ }^{4}$ Institute of Environmental and Operational Medicine, Tianjin 300050, People's Republic of China \\ ${ }^{5}$ Tianjin Key Laboratory of Environment, Nutrition and Public Health, Tianjin 300070, People's Republic of China \\ ${ }^{6}$ Center for International Collaborative Research on Environment, Nutrition and Public Health, Tianjin 300070, People's \\ Republic of China
}

(Submitted 1 February 2019 - Final revision received 7 September 2019 - Accepted 10 October 2019)

\section{Abstract}

Animal studies have suggested that mushroom intake can alleviate non-alcoholic fatty liver disease (NAFLD) due to its anti-inflammatory and antioxidant properties. However, the association between mushroom intake and NAFLD is unknown in humans. We aimed to investigate the association of mushroom intake with NAFLD among Chinese adults. This is a cross-sectional study of 24236 adults (mean (standard deviation) age: 40.7 (sD 11.9) years; 11394 men (47.0\%)). Mushroom intake was assessed via a validated FFQ. Newly diagnosed NAFLD was identified based on the results of annual health examinations, including ultrasound findings and a self-reported history of the disease. Multiple logistic models were used to examine the association between mushroom intake and NAFLD. The prevalence of newly diagnosed NAFLD was $19.0 \%$. Compared with those consuming mushrooms less frequently ( $\leq 1$ time/week), the fully adjusted OR of newly diagnosed NAFLD were 0.95 (95\% CI 0.86, 1.05) for those consuming 2-3 times/week and 0.76 (95\% CI 0.63, 0.92) for those consuming $\geq 4$ times/week $\left(P_{\text {for trend }}=0 \cdot 01\right)$. The inverse association was consistent in subgroups defined by age, sex and BMI. In conclusion, higher mushroom intake was significantly associated with lower prevalence of NAFLD among Chinese adults. Future research is required to understand the causal association between mushroom intake and NAFLD.

Key words: Mushrooms: Non-alcoholic fatty liver disease: Steatosis: Prevalence

Non-alcoholic fatty liver disease (NAFLD) is defined as the presence of more than $5 \%$ hepatic fat accumulation without other causes of chronic liver disease such as excessive alcohol consumption, hepatitis $\mathrm{C}$ or medications ${ }^{(1)}$. It has now become the most common liver disease, affecting at least $25-30 \%$ of adults in the general population and up to $70-90 \%$ of persons with obesity or type 2 diabetes $^{(2)}$. NAFLD may further progress to fibrosis, cirrhosis and even hepatocellular carcinoma ${ }^{(3)}$. In addition, NAFLD is associated with an increased risk of incident CVD and type 2 diabetes ${ }^{(4)}$. However, there is still no approved drug for the treatment of NAFLD. Therefore, lifestyle modifications consisting of diet, exercise and weight loss remain the main focuses of management in NAFLD ${ }^{(1)}$.

There has been a growing interest in the benefit of specific foods against NAFLD progression. Edible mushrooms are popular worldwide because of their taste and nutritional value. Mushrooms provide carbohydrate including fibre, protein and a range of vitamins (e.g. niacin), minerals and polyphenols ${ }^{(5,6)}$. Abundant evidence has suggested that mushrooms can counter inflammation $^{(7)}$ and oxidative stress ${ }^{(8)}$, which are major risk

Abbreviations: ALT, alanine aminotransferase; BP, blood pressure; FBG, fasting blood glucose; NAFLD, non-alcoholic fatty liver disease; PA, physical activity; TCLSIH, Tianjin Chronic Low-Grade Systemic Inflammation and Health.

* Corresponding author: Kaijun Niu, emails nkj0809@gmail.com; niukaijun@tmu.edu.cn

$\dagger$ These authors are the co-first authors and contributed equally to this work. 
factors involved in the pathogenesis of $\operatorname{NAFLD}^{(9,10)}$. Moreover, several animal studies have shown that mushroom intake can ameliorate NAFLD ${ }^{(11-13)}$. However, to date, the potential effects of habitual mushroom intake on NAFLD have not been evaluated in population-based studies.

Given that mushrooms are widely consumed in China and no epidemiological data are available on an association between mushroom consumption and NAFLD, the present study aimed to investigate an association between mushroom intake and NAFLD in the general population. We hypothesised that greater consumption of edible mushrooms is associated with a lower prevalence of NAFLD.

\section{Methods \\ Study design and participants}

The Tianjin Chronic Low-Grade Systemic Inflammation and Health (TCLSIH) Cohort Study is an ongoing prospective cohort study. Detailed descriptions of the TCLSIH Cohort Study have been published previously ${ }^{(14,15)}$. Briefly, the study was initiated in 2007 and all participants underwent a real-time ultrasonographic examination as part of health examinations since January 2010. Moreover, these participants were administered a detailed lifestyle questionnaire survey (including their dietary intake, physical activity (PA), household income, occupation, educational level, etc.) since May 2013. The study protocol was approved by the Institutional Review Board of Tianjin Medical University. All participants provided written informed consent.

For the present analysis, we used baseline data from the TCLSIH Cohort Study from 2013 to 2016 ( $n 32$ 165). We excluded subjects with a history of CVD ( $n$ 1552), cancer ( $n$ 248), alcoholic fatty liver disease ( $n$ 1099) or other liver diseases ( $n$ 124). Furthermore, we excluded subjects who were diagnosed with NAFLD from January 2010 to May 2013 or those with a selfreported history of the disease ( $n$ 4364), as they could have changed their eating habits after being diagnosed. Those who had missing data on dietary intake were also excluded ( $n$ 542). After these exclusions, this cross-sectional study consisted of 24236 participants (Fig. 1).

\section{Diagnosis of non-alcoholic fatty liver disease}

Fatty liver disease was diagnosed using abdominal ultrasonography, all unaware of the study aims. Abdominal ultrasonography was performed by experienced sonographers on a highresolution ultrasound machine (TOSHIBA SSA-660A; Toshiba) with a $2-5 \mathrm{MHz}$ curved array probe. Diagnosis of fatty liver disease required the presence of at least two of the following three abnormal findings ${ }^{(16)}$ : diffusely increased liver near-field ultrasound echo ('bright liver') and increased liver echotexture when compared with the kidneys, vascular blurring and deep attenuation of ultrasound signal. Participants with fatty liver disease and without significant alcohol consumption $(<140 \mathrm{~g} /$ week for men and $<70 \mathrm{~g} /$ week for women) were defined as having NAFLD $^{(17)}$. Furthermore, as a confirmatory analysis, participants who had NAFLD and elevated serum alanine aminotransferase

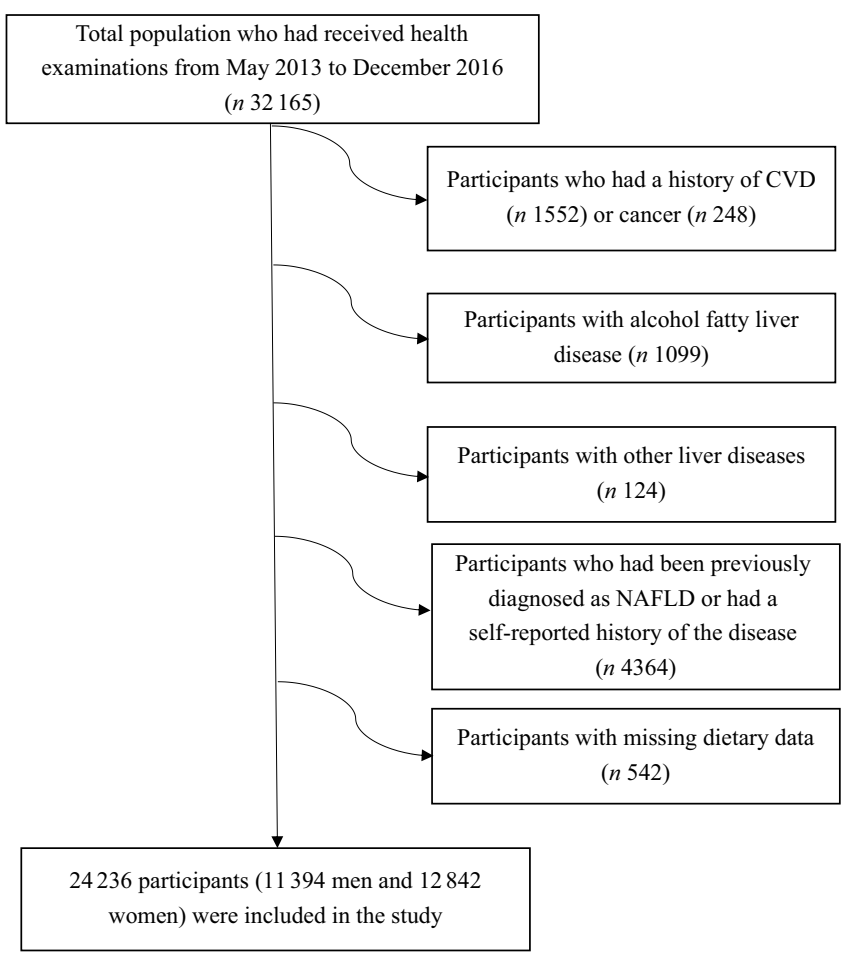

Fig. 1. Selection of subjects for the study. NAFLD, non-alcoholic fatty liver disease.

(ALT) levels (ALT >30 U/1 for men and >19 U/1 for women) were defined as having NAFLD with elevated $\operatorname{ALT}^{(18,19)}$.

\section{Assessment of dietary intake}

Diet was assessed by a self-administered FFQ that included 100 food items with specified serving sizes. The FFQ inquired about the mean consumption frequency of selected food items during the previous month using seven frequency categories ranging from 'almost never eat' to 'twice or more per day' for foods and eight frequency categories ranging from 'almost never drink' to 'four or more times per day' for beverages. The reproducibility and validity of the FFQ were assessed in a subsample of 150 participants from the TCLSIH study by comparing the data from two FFQ collected approximately 3 months apart and 4-d weighed dietary records including one weekend day. The Spearman's rank correlation coefficients comparing the FFQ to weighed dietary records were 0.49 for total energy, $0.35-0.54$ for nutrients ( $n$-3 fatty acid, fat and carbohydrate) and 0.69 for mushrooms. The Spearman correlation coefficients between two FFQ were $0 \cdot 68$ for total energy, 0.62-0.79 for food items (fruits, vegetables, sweet foods and beverages) and 0.75 for mushrooms. Energy and nutrient intakes were calculated by using an ad hoc computer programme developed to analyse the FFQ. Nutrientcomposition data for foods were based on the Chinese Food Composition Table ${ }^{(20)}$. Intakes of foods and nutrients were expressed as intakes per $2000 \mathrm{kcal}(8368 \mathrm{~kJ})$ of energy intake, calculated as: (food or nutrient intake/total energy intake) $\times$ $2000 \mathrm{kcal}(8368 \mathrm{~kJ})$.

Mushroom intake was assessed through one specific question. The question considered the consumption of edible mush- 
rooms, including Lentinula edodes, Auricularia auricula-judae, Pleurotus ostreatus, Flammulina velutipes, Agaricus bisporus and other. Participants answered the question using seven possible responses as follows: almost never, $<1$ time/week, 1 time/week, 2-3 times/week, 4-6 times/week, 1 time/d and $\geq 2$ times/d. In the present study, a typical serving size of mushrooms was 50 and $30 \mathrm{~g}$ for men and women, respectively. Mushroom consumption was categorised into three groups: $\leq 1$ time/week, $2-3$ times/week and $\geq 4$ times/week.

\section{Assessment of covariates}

Height and weight were measured by using standard protocols with participants wearing light clothing and no shoes. BMI was calculated as weight $(\mathrm{kg})$ divided by height squared $\left(\mathrm{m}^{2}\right)$. Waist circumference was measured to the nearest $0 \cdot 1 \mathrm{~cm}$ using a nonstretchable measuring tape at the umbilical level with subjects standing and breathing normally. Information on age (birthdate), sex, educational level, occupation, household income, smoking status, drinking status and medical disease was collected using a structured questionnaire.

Fasting blood samples were drawn $12 \mathrm{ml}$ from the cubital vein. Serum was used to measure fasting blood glucose (FBG) and lipids. FBG was measured using the glucose oxidase method, total cholesterol and TAG were measured using enzymatic methods, LDL was measured using the polyvinyl sulphuric acid precipitation method and HDL was measured using the chemical precipitation method. Serum ALT was measured using the International Federation of Clinical Chemistry method. These measurements were performed by using appropriate kits on a Cobas 8000 analyser (Roche).

After 5 min of rest, blood pressure (BP) was measured twice at the upper right arm using the TM-2655 oscillometric device (A\&D), while the participants were in a seated position. The mean of two readings was used for analysis. Hypertension was defined as systolic BP $\geq 140 \mathrm{mmHg}$ and/or diastolic BP $\geq 90$ mmHg or having a history of hypertension ${ }^{(21)}$. The metabolic syndrome was defined according to the criteria of the American Heart Association scientific statements of 2009 (22). PA in the most recent week was evaluated by using the short form of the International Physical Activity Questionnaire ${ }^{(23)}$. Total PA was calculated as metabolic equivalent hours per week (metabolic equivalent $\times \mathrm{h} /$ week).

\section{Statistical analysis}

To normalise the data, we used the natural logarithm transformation for all continuous variables. Baseline characteristics of participants were expressed as geometric means and 95\% CI for continuous variables and percentages for categorical variables. Participant characteristics were compared using ANCOVA for continuous variables and logistic regression analysis for categorical variables, with adjustments for age. Moreover, in Table 1, to evaluate whether the baseline variables increased or decreased with increasing mushroom consumption, we presented the $P$ values for linear trend.

We used logistic regression models to assess the association between mushroom intake and NAFLD or NAFLD with elevated ALT. The OR and corresponding 95\% CI were calculated using the $\leq 1$ time/week group as the reference. We fitted five models. Model 1 was a crude model. Model 2 was adjusted for age (continuous: years), sex (categorical: men and women) and BMI (continuous: $\mathrm{kg} / \mathrm{m}^{2}$ ). Model 3 was additionally adjusted for smoking status (categorical: current smoker, ex-smoker or non-smoker), drinking status (categorical: every-day drinker, sometime drinker, ex-drinker or non-drinker), educational level (categorical: $<$ or $\geq$ college graduate), occupation (categorical: managers, professionals and other), household income (categorical: $\leq$ or $>10000$ Yuan), PA (continuous: metabolic equivalent-h/week), family history of disease (including CVD, hypertension, hyperlipidaemia and diabetes (each yes or no)), individual history of disease (including hypertension, hyperlipidaemia and diabetes (each yes or no)) and total energy intake (continuous: $\mathrm{kcal} / \mathrm{d}$ ). Model 4 was further adjusted for FBG (continuous: $\mathrm{mmol} / \mathrm{l}$ ). Model 5 was adjusted for variables in model 4 plus niacin intake (quintiles) and three main dietary pattern scores (quintiles). Briefly, factor analysis with varimax rotation based on food items (mushrooms were not included in the calculation) was used to generate dietary patterns ${ }^{(24)}$. Three major dietary patterns were derived as follows: sweet pattern (higher factor loading of strawberry, kiwi fruit, persimmon, sweets, candied fruits, cakes, pineapple, cookies and ice cream), vegetable pattern (higher factor loading of celery, Chinese cabbage, cucumber, pumpkin, carrot, green vegetable, eggplant and raw vegetables) and animal food pattern (higher factor loading of animal organs, animal blood, preserved egg, sausage, instant noodle and seafood). Similar dietary patterns were derived in our previous study ${ }^{(24)}$. A higher dietary pattern score indicates stricter adherence to the dietary pattern. Moreover, $P_{\text {for trend }}$ values were calculated by using the categories of mushroom consumption ( $\leq 1$ time/week: 1; 2-3 times/week: 2 and $\geq 4$ times/week: 3 ) as an ordinal variable.

To assess effect modification, we calculated the $P$ values for interactions between mushroom consumption and all covariates through the inclusion of cross-product terms in the fully adjusted model. In addition, to test the robustness of our results, multicollinearity among all covariates was assessed using variance inflation factor. Variance inflation factor exceeding 10 was a sign of multicollinearity. Moreover, we also conducted subgroup analyses by age, sex and BMI.

All statistical analyses were performed by using SAS version 9.4 for Windows (SAS, Inc.). All statistical tests were two-sided, and a $P<0.05$ was considered to be statistically significant.

\section{Results}

Table 1 shows age-adjusted participant characteristics according to categories of mushroom consumption. Participants with higher mushroom intake tended to be younger and be more women and had lower BMI, waist circumference, TAG, diastolic BP, FBG, ALT and higher HDL. They were also more likely to be physically active, consumed more total energy and had higher sweet pattern score, vegetable pattern score and animal food pattern score. In addition, protein, carbohydrate, total fat, total vegetables, total fruits, sweet foods, EPA and DHA, white meat and niacin intake per $2000 \mathrm{kcal} / \mathrm{d}(8368 \mathrm{~kJ} / \mathrm{d})$ were higher in 
Table 1. Age-adjusted characteristics of the participants according to categories of mushroom consumption ( $n 24236)^{*}$ (Least square geometric mean values and $95 \%$ confidence intervals; percentages)

\begin{tabular}{|c|c|c|c|c|c|c|c|c|c|c|}
\hline \multirow[b]{3}{*}{ Characteristics } & \multicolumn{9}{|c|}{ Categories of mushroom consumption } & \multirow{3}{*}{$\begin{array}{c}P_{\text {for }} \\
\text { trend }\end{array}$} \\
\hline & \multicolumn{3}{|c|}{$\leq 1$ time/week ( $n$ 14 645) } & \multicolumn{3}{|c|}{ 2-3 times/week ( $n$ 7672) } & \multicolumn{3}{|c|}{$\geq 4$ times/week ( $n$ 1919) } & \\
\hline & Mean & $95 \% \mathrm{Cl}$ & $\%$ & Mean & $95 \% \mathrm{Cl}$ & $\%$ & Mean & $95 \% \mathrm{Cl}$ & $\%$ & \\
\hline Ageł (years) & 39.6 & $39 \cdot 4,39 \cdot 8$ & & $38 \cdot 2$ & $38 \cdot 0,38 \cdot 5$ & & $38 \cdot 4$ & $37 \cdot 9,38 \cdot 9$ & & $<0.0001$ \\
\hline Sex (men) & & & $49 \cdot 3$ & & & 44.7 & & & 38.8 & $<0.0001$ \\
\hline BMI $\left(\mathrm{kg} / \mathrm{m}^{2}\right)$ & 23.9 & $23 \cdot 9,24 \cdot 0$ & & 23.9 & $23 \cdot 8,24 \cdot 0$ & & 23.7 & $23 \cdot 5,23 \cdot 8$ & & $<0.01$ \\
\hline WC (cm) & $82 \cdot 2$ & $82 \cdot 0,82 \cdot 3$ & & $81 \cdot 8$ & $81 \cdot 6,82 \cdot 0$ & & $81 \cdot 1$ & $80.6,81.5$ & & $<0.0001$ \\
\hline $\mathrm{TC}(\mathrm{mmol} / \mathrm{l})$ & 4.63 & $4.62,4.64$ & & 4.64 & $4 \cdot 62,4.66$ & & 4.64 & $4.60,4.68$ & & 0.71 \\
\hline TAG $(\mathrm{mmol} / \mathrm{l})$ & 1.08 & $1.07,1.09$ & & 1.05 & $1.04,1.06$ & & 1.02 & $1.00,1.05$ & & $<0.0001$ \\
\hline LDL-cholesterol (mmol/l) & 2.68 & $2 \cdot 67,2 \cdot 69$ & & 2.68 & $2 \cdot 66,2 \cdot 69$ & & 2.67 & $2 \cdot 63,2 \cdot 70$ & & 0.52 \\
\hline HDL-cholesterol (mmol/l) & 1.35 & $1.34,1.36$ & & 1.37 & $1.36,1.38$ & & 1.40 & $1.38,1.42$ & & $<0.0001$ \\
\hline $\mathrm{SBP}(\mathrm{mmHg})$ & 118.5 & $118 \cdot 3,118 \cdot 7$ & & 118.4 & $118 \cdot 1,118.8$ & & $118 \cdot 2$ & $117 \cdot 6,118 \cdot 9$ & & 0.45 \\
\hline $\mathrm{DBP}(\mathrm{mmHg})$ & 74.5 & $74.4,74.7$ & & 74.2 & $74 \cdot 0,74 \cdot 4$ & & $74 \cdot 0$ & $73.5,74.5$ & & 0.03 \\
\hline $\mathrm{FBG}(\mathrm{mmol} / \mathrm{l})$ & 5.07 & $5.06,5.08$ & & 5.05 & $5.04,5.07$ & & 5.02 & $4.99,5.05$ & & $<0.01$ \\
\hline ALT (U/l) & $16 \cdot 9$ & $16 \cdot 7,17 \cdot 0$ & & 16.5 & $16 \cdot 2,16 \cdot 7$ & & $15 \cdot 8$ & $15 \cdot 3,16 \cdot 2$ & & $<0.0001$ \\
\hline PA (MET $\times$ h/week) & 9.29 & $9.09,9.49$ & & 11.4 & $11 \cdot 1,11 \cdot 7$ & & 11.8 & $11 \cdot 1,12 \cdot 5$ & & $<0.0001$ \\
\hline Total energy intake (kcal§/d) & $1878 \cdot 5$ & $\begin{array}{c}1869 \cdot 9 \\
1887 \cdot 1\end{array}$ & & $2127 \cdot 4$ & $\begin{array}{l}2114 \cdot 0 \\
2140 \cdot 8\end{array}$ & & $2336 \cdot 0$ & $\begin{array}{l}2306 \cdot 8 \\
2365 \cdot 6\end{array}$ & & $<0.0001$ \\
\hline Protein intake (g/2000 kcal $(8368 \mathrm{~kJ})$ per d) & $80 \cdot 2$ & $79.9,80 \cdot 6$ & & $87 \cdot 2$ & $86 \cdot 7,87 \cdot 7$ & & $105 \cdot 3$ & $104 \cdot 1,106 \cdot 4$ & & $<0.0001$ \\
\hline Carbohydrate intake (g/2000 kcal (8368 kJ) per d) & $344 \cdot 0$ & $342 \cdot 6,345 \cdot 5$ & & $368 \cdot 4$ & $366 \cdot 3,370 \cdot 6$ & & $447 \cdot 7$ & $442 \cdot 5,452 \cdot 8$ & & $<0.0001$ \\
\hline Total fat intake $(\mathrm{g} / 2000 \mathrm{kcal}(8368 \mathrm{~kJ})$ per d) & $43 \cdot 2$ & $43 \cdot 0,43.4$ & & $46 \cdot 5$ & $46 \cdot 2,46 \cdot 8$ & & 55.5 & $54 \cdot 7,56 \cdot 3$ & & $<0.0001$ \\
\hline Total vegetable intake $(\mathrm{g} / 2000 \mathrm{kcal}(8368 \mathrm{~kJ})$ per $\mathrm{d})$ & 215.5 & $213 \cdot 9,217 \cdot 1$ & & $285 \cdot 1$ & $282 \cdot 2,288 \cdot 0$ & & 409.5 & $401 \cdot 1,418 \cdot 0$ & & $<0.0001$ \\
\hline Total fruit intake $(\mathrm{g} / 2000 \mathrm{kcal}(8368 \mathrm{~kJ})$ per d) & 249.4 & $245 \cdot 6,253 \cdot 3$ & & $300 \cdot 7$ & $294.4,307 \cdot 2$ & & $380 \cdot 8$ & $365 \cdot 0,397 \cdot 2$ & & $<0.0001$ \\
\hline Sweet food intake $(\mathrm{g} / 2000 \mathrm{kcal}(8368 \mathrm{~kJ})$ per d) & $15 \cdot 8$ & $15 \cdot 4,16 \cdot 2$ & & 18.5 & $17 \cdot 9,19 \cdot 1$ & & $22 \cdot 7$ & $21 \cdot 2,24 \cdot 2$ & & $<0.0001$ \\
\hline EPA and DHA intake (g/2000 kcal (8368 kJ) per d) & 3.62 & $3.58,3.65$ & & 4.22 & $4 \cdot 16,4 \cdot 28$ & & 4.76 & $4.64,4.90$ & & $<0.0001$ \\
\hline Soft drink intake (ml/2000 kcal $(8368 \mathrm{~kJ})$ per d) & 4.97 & $4 \cdot 83,5 \cdot 11$ & & 4.85 & $4.66,5.05$ & & 5.04 & $4.66,5.46$ & & 0.73 \\
\hline Red meat intake $(\mathrm{g} / 2000 \mathrm{kcal}(8368 \mathrm{~kJ})$ per d) & 23.5 & $23 \cdot 1,23 \cdot 9$ & & $25 \cdot 1$ & $24 \cdot 4,25 \cdot 7$ & & $23 \cdot 0$ & $21 \cdot 9,24 \cdot 1$ & & 0.43 \\
\hline White meat intake $(\mathrm{g} / 2000 \mathrm{kcal}(8368 \mathrm{~kJ})$ per d) & $16 \cdot 6$ & $16 \cdot 3,16 \cdot 9$ & & $19 \cdot 0$ & $18 \cdot 6,19.5$ & & $19 \cdot 1$ & $18 \cdot 2,20 \cdot 1$ & & $<0.0001$ \\
\hline Niacin intake (mg/2000 kcal $(8368$ kJ) per d) & $22 \cdot 1$ & $22 \cdot 0,22 \cdot 2$ & & 24.1 & $23 \cdot 9,24 \cdot 2$ & & 29.2 & $28 \cdot 9,29 \cdot 5$ & & $<0.0001$ \\
\hline Sweet dietary pattern score & -0.11 & $-0.13,-0.09$ & & 0.07 & $0.05,0.09$ & & 0.55 & $0.51,0.59$ & & $<0.0001$ \\
\hline Healthy dietary pattern score & -0.27 & $-0.28,-0.25$ & & 0.23 & $0.21,0.25$ & & $1 \cdot 15$ & $1 \cdot 11,1 \cdot 19$ & & $<0.0001$ \\
\hline Animal food dietary pattern score & -0.05 & $-0.06,-0.03$ & & 0.00 & $-0.02,0.02$ & & 0.36 & $0.31,0.40$ & & $<0.0001$ \\
\hline \multicolumn{11}{|l|}{ Smoking status } \\
\hline Smoker & & & 19.4 & & & $15 \cdot 3$ & & & 12.5 & $<0.0001$ \\
\hline Ex-smoker & & & 4.66 & & & 4.60 & & & 3.71 & 0.45 \\
\hline Non-smoker & & & 75.9 & & & $80 \cdot 1$ & & & 83.8 & $<0.0001$ \\
\hline \multicolumn{11}{|l|}{ Drinking status } \\
\hline Every-day & & & 3.73 & & & 2.93 & & & 3.42 & 0.10 \\
\hline Sometime & & & $55 \cdot 0$ & & & $55 \cdot 5$ & & & $50 \cdot 8$ & 0.04 \\
\hline Ex-drinker & & & 9.98 & & & $10 \cdot 2$ & & & 11.5 & 0.15 \\
\hline Non-drinker & & & 31.3 & & & 31.4 & & & 34.3 & 0.04 \\
\hline Education level (college or higher) & & & $64 \cdot 2$ & & & $69 \cdot 4$ & & & $66 \cdot 3$ & $<0.01$ \\
\hline \multicolumn{11}{|l|}{ Occupation } \\
\hline Managers & & & $40 \cdot 6$ & & & 43.3 & & & $42 \cdot 8$ & $<0.01$ \\
\hline Professionals & & & $16 \cdot 8$ & & & $16 \cdot 8$ & & & $15 \cdot 7$ & 0.19 \\
\hline Other & & & $42 \cdot 6$ & & & $40 \cdot 0$ & & & 41.6 & 0.046 \\
\hline Household income ( $\geq 10000$ Yuan) & & & 33.4 & & & $37 \cdot 4$ & & & 33.9 & $<0.001$ \\
\hline Metabolic syndrome (yes) & & & $19 \cdot 9$ & & & $18 \cdot 3$ & & & $15 \cdot 9$ & $<0.01$ \\
\hline Hypertension & & & $19 \cdot 9$ & & & $17 \cdot 8$ & & & $17 \cdot 8$ & 0.08 \\
\hline Hyperlipidaemia & & & $40 \cdot 1$ & & & 38.0 & & & 37.6 & 0.40 \\
\hline Diabetes & & & 3.09 & & & 2.45 & & & 2.34 & 0.02 \\
\hline \multicolumn{11}{|l|}{ Family history of disease } \\
\hline CVD & & & $27 \cdot 4$ & & & $26 \cdot 6$ & & & $26 \cdot 0$ & 0.81 \\
\hline Hypertension & & & $47 \cdot 0$ & & & 48.9 & & & 44.4 & 0.35 \\
\hline Hyperlipidaemia & & & 0.23 & & & 0.23 & & & 0.16 & 0.71 \\
\hline Diabetes & & & 23.9 & & & 23.8 & & & 24.4 & 0.70 \\
\hline
\end{tabular}

WC, waist circumference; TC, total cholesterol; SBP, systolic blood pressure; DBP, diastolic blood pressure; FBG, fasting blood glucose; ALT, alanine aminotransferase; PA, physical activity; MET, metabolic equivalent.

* Continuous variables are expressed as least square geometric means and $95 \% \mathrm{Cl}$ and categorical variables are expressed as percentages.

$\dagger$ ANCOVA or logistic regression analysis adjusted for age where appropriate. The $P_{\text {for trend }}$ values were calculated by using the categories of mushroom consumption

( $\leq 1$ time/week: 1 ; 2-3 times/week: 2 and $\geq 4$ times/week: 3 ) as an ordinal variable.

$\ddagger$ Values were not age-adjusted.

$\S$ To convert kcal to kJ, multiply by $4 \cdot 184$. 
Table 2. Age-adjusted characteristics of the participants by non-alcoholic fatty liver disease (NAFLD) status $(n 24236)^{*}$ (Least square geometric mean values and $95 \%$ confidence intervals; percentages)

\begin{tabular}{|c|c|c|c|c|c|c|c|}
\hline \multirow[b]{3}{*}{ Characteristics } & \multicolumn{6}{|c|}{ NAFLD status } & \multirow[b]{3}{*}{$P \dagger$} \\
\hline & \multicolumn{3}{|c|}{ No $(n 19631)$} & \multicolumn{3}{|c|}{ Yes ( $n$ 4605) } & \\
\hline & Mean & $95 \% \mathrm{Cl}$ & $\%$ & Mean & $95 \% \mathrm{Cl}$ & $\%$ & \\
\hline Age $\neq$ (years) & $38 \cdot 4$ & $38 \cdot 3,38 \cdot 6$ & & $42 \cdot 1$ & $41 \cdot 8,42 \cdot 5$ & & $<0.0001$ \\
\hline Sex (men) & & & $41 \cdot 3$ & & & $71 \cdot 6$ & $<0.0001$ \\
\hline BMI $\left(\mathrm{kg} / \mathrm{m}^{2}\right)$ & $23 \cdot 1$ & $23 \cdot 1,23 \cdot 1$ & & $27 \cdot 6$ & $27 \cdot 5,27 \cdot 7$ & & $<0.0001$ \\
\hline WC $(\mathrm{cm})$ & $79 \cdot 7$ & $79 \cdot 6,79 \cdot 8$ & & $92 \cdot 2$ & $91 \cdot 9,92 \cdot 5$ & & $<0.0001$ \\
\hline $\mathrm{TC}(\mathrm{mmol} / \mathrm{l})$ & 4.58 & $4.57,4.59$ & & 4.85 & $4.83,4.88$ & & $<0.0001$ \\
\hline TAG (mmol/l) & 0.96 & $0.95,0.97$ & & 1.65 & $1.62,1.67$ & & $<0.0001$ \\
\hline LDL-cholesterol (mmol/l) & 2.59 & $2.58,2.60$ & & $2 \cdot 87$ & $2 \cdot 85,2 \cdot 89$ & & $<0.0001$ \\
\hline HDL-cholesterol (mmol//) & 1.43 & $1.43,1.44$ & & $1 \cdot 11$ & $1 \cdot 10,1 \cdot 12$ & & $<0.0001$ \\
\hline $\mathrm{SBP}(\mathrm{mmHg})$ & $116 \cdot 4$ & $116 \cdot 2,116 \cdot 6$ & & 127.4 & $127,127 \cdot 9$ & & $<0.0001$ \\
\hline $\mathrm{DBP}(\mathrm{mmHg})$ & 73.0 & $72 \cdot 9,73 \cdot 1$ & & $80 \cdot 5$ & $80 \cdot 2,80 \cdot 8$ & & $<0.0001$ \\
\hline $\mathrm{FBG}(\mathrm{mmol} / \mathrm{l})$ & 4.98 & $4.98,4.99$ & & $5 \cdot 39$ & $5 \cdot 37,5 \cdot 41$ & & $<0.0001$ \\
\hline $\operatorname{ALT}(\mathrm{U} / \mathrm{I})$ & $14 \cdot 7$ & $14 \cdot 6,14 \cdot 8$ & & $26 \cdot 9$ & $26 \cdot 5,27 \cdot 3$ & & $<0.0001$ \\
\hline PA (MET $\times$ h/week) & 9.96 & $9 \cdot 78,10 \cdot 2$ & & $10 \cdot 7$ & $10 \cdot 3,11 \cdot 1$ & & $<0.01$ \\
\hline Total energy intake (kcal§/d) & $1987 \cdot 2$ & $1979 \cdot 1,1995 \cdot 3$ & & 1991.4 & $1974 \cdot 6,2008 \cdot 3$ & & 0.66 \\
\hline Protein intake (g/2000 kcal $(8368 \mathrm{~kJ})$ per d) & 83.7 & $83 \cdot 4,84 \cdot 0$ & & $86 \cdot 1$ & $85 \cdot 4,86 \cdot 7$ & & $<0.0001$ \\
\hline Carbohydrate intake (g/2000 kcal $(8368 \mathrm{~kJ})$ per d) & 359.4 & $358 \cdot 1,360 \cdot 7$ & & $357 \cdot 2$ & $354.5,360 \cdot 0$ & & 0.17 \\
\hline Total fat intake (g/2000 kcal (8368 kJ) per d) & $44 \cdot 7$ & $44.5,44.9$ & & $47 \cdot 0$ & $46 \cdot 5,47 \cdot 4$ & & $<0.0001$ \\
\hline Total vegetable intake $(\mathrm{g} / 2000 \mathrm{kcal}(8368 \mathrm{~kJ})$ per $\mathrm{d})$ & 248.5 & $246 \cdot 8,250 \cdot 3$ & & 244.4 & $240 \cdot 9,248 \cdot 0$ & & 0.04 \\
\hline Total fruit intake $(\mathrm{g} / 2000 \mathrm{kcal}(8368 \mathrm{~kJ})$ per d) & 275.8 & $272 \cdot 1,279.5$ & & 264.8 & $257 \cdot 5,272 \cdot 2$ & & $<0.01$ \\
\hline Sweet food intake (g/2000 kcal $(8368 \mathrm{~kJ})$ per d) & $17 \cdot 6$ & $17 \cdot 2,18 \cdot 0$ & & $15 \cdot 1$ & $14 \cdot 5,15 \cdot 7$ & & $<0.0001$ \\
\hline EPA and DHA intake (g/2000 kcal $(8368 \mathrm{~kJ})$ per d) & 3.86 & $3.82,3.89$ & & 4.00 & $3.93,4.07$ & & $<0.001$ \\
\hline Soft drink intake (ml/2000 kcal (8368 kJ) per d) & 4.63 & $4.52,4.75$ & & 6.49 & $6 \cdot 17,6 \cdot 84$ & & $<0.0001$ \\
\hline Red meat intake (g/2000 kcal $(8368 \mathrm{~kJ})$ per d) & $23 \cdot 2$ & $22 \cdot 9,23 \cdot 6$ & & $27 \cdot 1$ & $26 \cdot 3,28 \cdot 0$ & & $<0.0001$ \\
\hline White meat intake $(\mathrm{g} / 2000 \mathrm{kcal}(8368 \mathrm{~kJ})$ per d) & $17 \cdot 1$ & $16 \cdot 8,17 \cdot 4$ & & $19 \cdot 4$ & $18 \cdot 8,20 \cdot 1$ & & $<0.0001$ \\
\hline Niacin intake $(\mathrm{mg} / 2000 \mathrm{kcal}(8368 \mathrm{~kJ})$ per d) & $23 \cdot 1$ & $23 \cdot 1,23 \cdot 2$ & & 23.5 & $23 \cdot 3,23 \cdot 7$ & & 0.001 \\
\hline Sweet dietary pattern score & 0.00 & $-0.01,0.02$ & & -0.02 & $-0.05,0.01$ & & 0.20 \\
\hline Healthy dietary pattern score & 0.00 & $-0.01,0.02$ & & -0.02 & $-0.05,0.01$ & & 0.20 \\
\hline Animal food dietary pattern score & -0.01 & $-0.03,0.00$ & & 0.05 & $0.02,0.08$ & & $<0.0001$ \\
\hline \multicolumn{8}{|l|}{ Smoking status } \\
\hline Smoker & & & $15 \cdot 2$ & & & $28 \cdot 2$ & $<0.0001$ \\
\hline Ex-smoker & & & 4.02 & & & 6.99 & $<0.0001$ \\
\hline Non-smoker & & & $80 \cdot 8$ & & & 64.8 & $<0.0001$ \\
\hline \multicolumn{8}{|l|}{ Drinking status } \\
\hline Every-day & & & 3.78 & & & 2.06 & $<0.0001$ \\
\hline Sometime & & & $53 \cdot 1$ & & & $62 \cdot 2$ & $<0.0001$ \\
\hline Ex-drinker & & & $9 \cdot 77$ & & & $11 \cdot 8$ & $<0.0001$ \\
\hline Non-drinker & & & 33.4 & & & $24 \cdot 0$ & $<0.0001$ \\
\hline Education level (college or higher) & & & $68 \cdot 0$ & & & $57 \cdot 6$ & $<0.0001$ \\
\hline \multicolumn{8}{|l|}{ Occupation } \\
\hline Managers & & & $42 \cdot 5$ & & & 37.9 & $<0.0001$ \\
\hline Professionals & & & $16 \cdot 6$ & & & $17 \cdot 2$ & 0.03 \\
\hline Other & & & $41 \cdot 0$ & & & 44.9 & $<0.01$ \\
\hline Household income ( $\geq 10000$ Yuan) & & & $35 \cdot 0$ & & & 33.3 & 0.12 \\
\hline Metabolic syndrome (yes) & & & $10 \cdot 6$ & & & $55 \cdot 1$ & $<0.0001$ \\
\hline Hypertension & & & 13.9 & & & $41 \cdot 1$ & $<0.0001$ \\
\hline Hyperlipidaemia & & & $32 \cdot 7$ & & & $67 \cdot 1$ & $<0.0001$ \\
\hline Diabetes & & & 1.50 & & & 8.49 & $<0.0001$ \\
\hline \multicolumn{8}{|l|}{ Family history of disease } \\
\hline CVD & & & $27 \cdot 6$ & & & 24.7 & $<0.0001$ \\
\hline Hypertension & & & $47 \cdot 1$ & & & $48 \cdot 3$ & 0.47 \\
\hline Hyperlipidaemia & & & 0.28 & & & 0.02 & $<0.01$ \\
\hline Diabetes & & & $23 \cdot 0$ & & & $28 \cdot 0$ & $<0.0001$ \\
\hline
\end{tabular}

WC, waist circumference; TC, total cholesterol; SBP, systolic blood pressure; DBP, diastolic blood pressure; FBG, fasting blood glucose; ALT, alanine aminotransferase; PA, physical activity; MET, metabolic equivalent.

* Continuous variables are expressed as least square geometric means and $95 \% \mathrm{Cl}$ and categorical variables are expressed as percentages.

† ANCOVA or logistic regression analysis adjusted for age where appropriate.

¥ Values were not age-adjusted.

$\S$ To convert kcal to kJ, multiply by $4 \cdot 184$.

people with increased mushroom consumption. Furthermore, those with higher mushroom intake were more likely to be non-smokers and non-drinkers or employed as managers, and they were less likely to be occasional drinkers and had higher educational level and household income, and they had a lower prevalence of the metabolic syndrome and diabetes.

Table 2 shows age-adjusted participant characteristics by NAFLD status. Compared with participants without NAFLD, those 
Table 3. Association of mushroom consumption with non-alcoholic fatty liver disease (NAFLD) or NAFLD with elevated alanine aminotransferase (ALT) ( $n$ 24 236)

(Odds ratios and $95 \%$ confidence intervals)

\begin{tabular}{|c|c|c|c|c|}
\hline \multirow[b]{2}{*}{ Logistic regression models } & \multicolumn{3}{|c|}{ Categories of mushroom consumption } & \multirow[b]{2}{*}{$P_{\text {for trend }}{ }^{*}$} \\
\hline & $\leq 1$ time/week & 2-3 times/week & $\geq 4$ times/week & \\
\hline No. of subjects & 14645 & 7672 & 1919 & - \\
\hline \multirow{2}{*}{\multicolumn{4}{|c|}{$\begin{array}{l}\text { No. of NAFLD } \\
\text { Model } 1\end{array}$}} & - \\
\hline & & & & $<00001$ \\
\hline OR & 1.00 & 0.87 & 0.70 & \\
\hline $95 \% \mathrm{Cl}$ & Reference & $0.81,0.94$ & $0.62,0.80$ & \\
\hline \multicolumn{4}{|l|}{ Model 2} & $<0.0001$ \\
\hline OR & 1.00 & 0.90 & $0 \cdot 70$ & \\
\hline $95 \% \mathrm{Cl}$ & Reference & $0.83,0.99$ & $0.59,0.83$ & \\
\hline \multicolumn{4}{|l|}{ Model 3} & $<0.0001$ \\
\hline OR & 1.00 & 0.91 & 0.70 & \\
\hline $95 \% \mathrm{Cl}$ & Reference & $0.83,1.00$ & $0.59,0.83$ & \\
\hline \multicolumn{4}{|l|}{ Model 4} & $<0.0001$ \\
\hline OR & 1.00 & 0.91 & 0.70 & \\
\hline $95 \% \mathrm{Cl}$ & Reference & $0.83,1.00$ & $0.58,0.83$ & \\
\hline \multicolumn{4}{|l|}{ Model 5} & 0.01 \\
\hline OR & 1.00 & 0.95 & 0.76 & \\
\hline $95 \% \mathrm{Cl}$ & Reference & $0.86,1.05$ & $0.63,0.92$ & \\
\hline \multirow{2}{*}{\multicolumn{4}{|c|}{$\begin{array}{l}\text { No. of NAFLD with elevated ALT } \\
\text { Model } 1\end{array}$}} & - \\
\hline & & & & $<0001$ \\
\hline OR & 1.00 & $0.89 \dagger$ & 0.75 & \\
\hline $95 \% \mathrm{Cl}$ & Reference & $0.81,0.98$ & $0.62,0.89$ & \\
\hline \multicolumn{4}{|l|}{ Model 2} & $<0.01$ \\
\hline OR & 1.00 & 0.89 & 0.76 & \\
\hline $95 \% \mathrm{Cl}$ & Reference & $0.80,1.00$ & $0.62,0.94$ & \\
\hline \multicolumn{4}{|l|}{ Model 3} & $<0.01$ \\
\hline OR & 1.00 & 0.90 & 0.75 & \\
\hline $95 \% \mathrm{Cl}$ & Reference & $0.80,1.02$ & $0.60,0.93$ & \\
\hline \multicolumn{4}{|l|}{ Model 4} & $<0.01$ \\
\hline OR & 1.00 & 0.90 & 0.76 & \\
\hline $95 \% \mathrm{Cl}$ & Reference & $0.80,1.02$ & $0.61,0.94$ & \\
\hline \multicolumn{4}{|l|}{ Model 5} & 0.03 \\
\hline OR & 1.00 & 0.92 & 0.78 & \\
\hline $95 \% \mathrm{Cl}$ & Reference & $0.81,1.04$ & $0.62,0.98$ & \\
\hline \multicolumn{5}{|c|}{ 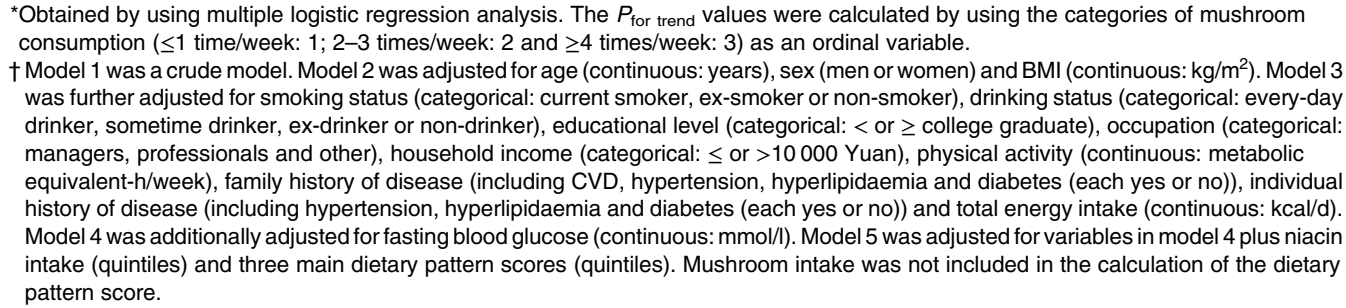 } \\
\hline
\end{tabular}

with NAFLD tended to be older, men and to have higher BMI, waist circumference, total cholesterol, TAG, LDL, systolic BP, diastolic BP, FBG, ALT, PA, animal food pattern score and lower HDL. They also had higher protein, total fat, EPA and DHA, soft drinks, red meat, white meat and niacin intakes per $2000 \mathrm{kcal} / \mathrm{d}$ $(8368 \mathrm{~kJ} / \mathrm{d})$, but had lower vegetable, fruit and sweet food intakes per $2000 \mathrm{kcal} / \mathrm{d}(8368 \mathrm{~kJ} / \mathrm{d})$. In addition, a higher proportion of these participants was current smokers or ex-smokers, sometime drinkers or ex-drinkers, employed as professionals or other and had the metabolic syndrome, hypertension, hyperlipidaemia, diabetes and family history of diabetes. A lower proportion of these participants was non-smokers, every-day drinkers, non-drinkers, with higher educational level, employed as managers and had family history of CVD or hyperlipidaemia.

Table 3 presents the crude and adjusted associations between categories of mushroom consumption and newly diagnosed
NAFLD or NAFLD with elevated ALT. The OR for newly diagnosed NAFLD decreased across increasing frequency of mushroom consumption. After adjustment for age, sex and BMI, the OR of newly diagnosed NAFLD across categories of mushroom consumption were 1.00 (reference) for $\leq 1$ time/week, $0.90(95 \%$ CI 0.83, 0.99) for 2-3 times/week and 0.70 (95\% CI 0.59, 0.83) for $\geq 4$ times/week $\left(P_{\text {for trend }}<0.0001\right)$. Additional adjustments for sociodemographic and lifestyle factors as well as for FBG did not nearly change the inverse association. Further adjustments for dietary niacin intake and three major dietary patterns made the association slightly attenuated; the fully adjusted OR of newly diagnosed NAFLD were 1.00 (reference) for $\leq 1$ time/week, 0.95 (95\% CI 0.86, 1.05) for 2-3 times/week and 0.76 (95\% CI 0.63, 0.92 ) for $\geq 4$ times/week $\left(P_{\text {for trend }}=0 \cdot 01\right.$ ). Moreover, similar associations were also observed for NAFLD with elevated ALT. The fully adjusted OR of NAFLD with elevated ALT were 
1.00 (reference) for $\leq 1$ time/week, 0.92 (95\% CI 0.81, 1.04) for 2-3 times/week and 0.78 (95\% CI 0.62, 0.98) for $\geq 4$ times/week $\left(P_{\text {for trend }}=0.03\right)$.

There was no evidence in the final model that the covariates modified the effect of the association between mushroom consumption and NAFLD (all $P$ for interaction $\geq 0 \cdot 10$ ). For multicollinearity, all variance inflation factor values ranged from 1.01 to $2 \cdot 44$, indicating that no collinearity was accepted. In addition, the inverse association between mushroom intake and NAFLD persisted in subgroup analyses (data not shown).

\section{Discussion}

This is the first large population-based study to investigate the association between edible mushroom intake and NAFLD. The results suggested that high mushroom intake was associated with a low prevalence of NAFLD in the general Chinese population.

In the present study, we adjusted for a considerable number of confounding factors. First, we adjusted for age, sex and BMI. However, the results hardly changed after adjustment. Therefore, high mushroom intake was associated with a low prevalence of NAFLD, independent of age, sex and BMI. Second, sociodemographic variables, lifestyle factors, medical history and total energy intake ${ }^{(25)}$ are closely associated with NAFLD. Therefore, we further adjusted for smoking status, drinking status, educational level, occupation, household income, PA, family history of disease, individual history of disease and total energy intake. However, the inverse association remained statistically significant when adjusted for these factors. Third, NAFLD is strongly associated with insulin resistance. Therefore, we further adjusted for FBG as a biomarker of insulin resistance. However, the inverse association between mushroom intake and NAFLD did not substantially change after adjustment for FBG. These results suggested that the impact of mushroom intake on NAFLD was independent of mediation by protection against insulin resistance. Finally, compared with individual nutrients or foods, dietary patterns represent a broader picture of nutrient and food consumption. It can be used as a covariate when examining a specific food, to determine whether the effect of the food is independent of the overall $\operatorname{diet}^{(26)}$. Furthermore, a recent study has shown that high dietary niacin intake may have a favourable effect on the reduction of liver fat ${ }^{(27)}$. Studies also have demonstrated that mushrooms contain high amounts of niacin ${ }^{(6)}$. Thus, in a final model, we further adjusted for three major dietary patterns and dietary niacin intake. However, the inverse association between mushroom consumption and NAFLD remained significant when adjusted for these dietary factors. These results indicated that the effect of mushroom on NAFLD is independent of niacin intake and overall diet.

A study conducted in mice demonstrated that $10 \%$ Agaricus bisporus supplementation can reduce high-fat diet-induced NAFLD development after feeding 10 weeks $^{(11)}$. Similarly, another animal study also showed that dietary supplementation with $10 \%$ air-dried powder of Mukitake mushrooms can alleviate NAFLD after an 8-week feeding period ${ }^{(12)}$. These results from animal studies suggest that mushroom intake could have beneficial effects for mice with NAFLD. However, the association between mushroom consumption and NAFLD in humans remains unknown. The data from our human studies have been consistent with those of the animal studies. Therefore, our present study provides novel data that support a beneficial association between higher mushroom intake and a lower prevalence of NAFLD in the general population.

Many people in China consume a variety of edible mushrooms daily ${ }^{(28)}$. Mushrooms contain many nutritional components such as polyphenols, vitamins, minerals, fibres and polysaccharides $^{(29)}$. Studies have suggested that edible mushrooms are potent antioxidants and anti-inflammatory agents $(7,8)$ that could play important roles in the development of NAFLD $^{(10,30)}$. Moreover, an animal study have indicated that supplementation with a variety of mushrooms (containing a 0.5 or $3 \%$ mushroom mixture, which was equivalent to approximately 100 or $600 \mathrm{~g}$ of fresh mushroom intake per $\mathrm{d}$ in humans) can suppress visceral fat accumulation and improve gut microbiota $^{(13)}$. Furthermore, insulin resistance plays an important role in the pathogenesis of NAFLD. A randomised clinical trial showed that mushroom extract supplements (containing $500 \mathrm{mg}$ Agaricus blazei Murill extract three times daily for 12 weeks) could improve insulin resistance ${ }^{(31)}$. Additionally, mushroom intake could suppress appetites, thereby reducing food intake ${ }^{(32)}$. Therefore, the inverse association between mushroom intake and NAFLD development is biologically reasonable.

Notably, Table 2 shows NAFLD patients are more physically active than controls. Because men (geometric mean of PA was $11.8(95 \%$ CI $11.5,12 \cdot 1)$ ) were more physically active than women (geometric mean of PA was 8.82 (95\% CI 8.62, 9.02)), we consider sex is an important confounding factor that explains the fact that NAFLD patients are more physically active than controls. Accordingly, we adjusted for age and sex. The results showed that age- and sex-adjusted geometric mean of PA was $10 \cdot 2$ (95\% CI 10.0, 10.4) in controls and 10.1 (95\% CI 9.68, $10 \cdot 5)$ in NAFLD patients $(P=0.50)$. Therefore, the fact that NAFLD patients are more physically active than controls was confounded by sex.

To our knowledge, this is the first study that examined the association between mushroom consumption and NAFLD in humans. Strengths of our study include a large sample size and detailed measurement of habitual diet with a validated FFQ. Moreover, detailed information about lifestyle factors enabled us to conduct comprehensive analyses by taking into account many confounding factors.

The present study has several limitations. First, NAFLD was diagnosed using ultrasonography rather than liver biopsy (the 'gold standard' for diagnosing NAFLD). However, liver biopsy is time-consuming and expensive and not feasible in large observational studies. Second, diet was measured using an FFQ. Although validated, FFQ are subject to misclassification and random error. Therefore, the association was attenuated between mushroom intake and NAFLD. Third, high mushroom intake may be a marker of other healthy lifestyle factors. Baseline participant characteristics of the TCLSIH Cohort 
Study (Table 1) and Ohsaki Cohort 2006 Study $^{(33)}$ show that participants with higher mushroom intake had many healthier dietary behaviours and lifestyle characteristics such as higher vegetable and fruit intake, less smoking and drinking and more physically active. However, adjustments for these factors did not substantially change the inverse association. Fourth, genetic and epigenetic factors play an important role in the pathogenesis of NAFLD ${ }^{(34,35)}$. Therefore, our results may not be generalisable to other populations. Finally, although we carefully adjusted for many potential confounding factors, there are likely to remain potentially important confounding differences that could affect the results ${ }^{(36)}$.

\section{Conclusions}

In conclusion, the present study provides the first evidence of an association between edible mushrooms and NAFLD in humans. Further prospective cohort studies are needed to validate these results.

\section{Acknowledgements}

The authors gratefully acknowledge all the people that have made the present study.

The present study was supported by grants from the National Natural Science Foundation of China (no. 91746205).

S. Z. analysed data and wrote the paper. Y. G., M. L., J. F., Q. Z., L. L., G. M., Z. Y., H. W., X. B., S. S., X. W., M. Z., Q. J., K. S. and Y. W. conducted research. K. N. designed research and had primary responsibility for final content. All authors read and approved the final manuscript.

There are no conflicts of interest.

\section{References}

1. Chalasani N, Younossi Z, Lavine JE, et al. (2018) The diagnosis and management of nonalcoholic fatty liver disease: practice guidance from the American association for the study of liver diseases. Hepatology 67, 328-357.

2. Younossi ZM, Koenig AB, Abdelatif D, et al. (2016) Global epidemiology of nonalcoholic fatty liver disease-meta-analytic assessment of prevalence, incidence, and outcomes. Hepatology 64, 73-84.

3. Vernon G, Baranova A \& Younossi ZM (2011) Systematic review: the epidemiology and natural history of non-alcoholic fatty liver disease and non-alcoholic steatohepatitis in adults. Aliment Pharmacol Ther 34, 274-285.

4. Morrison AE, Zaccardi F, Khunti K, et al. (2018) Causality between non-alcoholic fatty liver disease and risk of cardiovascular disease and type 2 diabetes: a meta-analysis with bias analysis. Liver Int 39, 557-567.

5. Zhang JJ, Li Y, Zhou T, et al. (2016) Bioactivities and health benefits of mushrooms mainly from China. Molecules 21, pii: E938.

6. Wang XM, Zhang J, Wu LH, et al. (2014) A mini-review of chemical composition and nutritional value of edible wildgrown mushroom from China. Food Chem 151, 279-285.

7. Muszynska B, Grzywacz-Kisielewska A, Kala K, et al. (2018) Anti-inflammatory properties of edible mushrooms: a review. Food Chem 243, 373-381.

8. Kozarski M, Klaus A, Jakovljevic D, et al. (2015) Antioxidants of edible mushrooms. Molecules 20, 19489-19525.
9. Al Rifai M, Silverman MG, Nasir K, et al. (2015) The association of nonalcoholic fatty liver disease, obesity, and metabolic syndrome, with systemic inflammation and subclinical atherosclerosis: the Multi-Ethnic Study of Atherosclerosis (MESA). Atherosclerosis 239, 629-633.

10. Masarone M, Rosato V, Dallio M, et al. (2018) Role of oxidative stress in pathophysiology of nonalcoholic fatty liver disease. Oxid Med Cell Longev 2018, 9547613.

11. Iniguez M, Perez-Matute P, Villanueva-Millan MJ, et al. (2018) Agaricus bisporus supplementation reduces high-fat dietinduced body weight gain and fatty liver development. $J$ Physiol Biochem 74, 635-646.

12. Nagao K, Inoue N, Inafuku M, et al. (2010) Mukitake mushroom (Panellus serotinus) alleviates nonalcoholic fatty liver disease through the suppression of monocyte chemoattractant protein 1 production in $\mathrm{db} / \mathrm{db}$ mice. J Nutr Biochem 21, 418-423.

13. Shimizu T, Mori K, Ouchi K, et al. (2018) Effects of dietary intake of Japanese mushrooms on visceral fat accumulation and gut microbiota in Mice. Nutrients 10, pii: E610.

14. Gu Y, Li H, Bao X, et al. (2017) The relationship between Thyroid function and the prevalence of type 2 diabetes mellitus in Euthyroid subjects. J Clin Endocrinol Metab 102, 434-442.

15. Wang H, Gu Y, Zheng L, et al. (2018) Association between bedtime and the prevalence of newly diagnosed non-alcoholic fatty liver disease in adults. Liver Int 38, 2277-2286.

16. Farrell GC, Chitturi S, Lau GK, et al. (2007) Guidelines for the assessment and management of non-alcoholic fatty liver disease in the Asia-Pacific region: executive summary. $J$ Gastroenterol Hepatol 22, 775-777.

17. Gao X, Fan JG \& Study Group of Liver and Metabolism, Chinese Society of Endocrinology. (2013) Diagnosis and management of non-alcoholic fatty liver disease and related metabolic disorders: consensus statement from the Study Group of Liver and Metabolism, Chinese Society of Endocrinology. J Diabetes 5 , 406-415.

18. Prati D, Taioli E, Zanella A, et al. (2002) Updated definitions of healthy ranges for serum alanine aminotransferase levels. Ann Intern Med 137, 1-10.

19. Ruhl CE \& Everhart JE (2009) Elevated serum alanine aminotransferase and gamma-glutamyltransferase and mortality in the United States population. Gastroenterology 136, 477-485.

20. Yang Y (2009) China Food Composition, 2nd ed. Beijing, China: Peking University Medical Press.

21. Chobanian AV, Bakris GL, Black HR, et al. (2003) The seventh report of the joint national committee on prevention, detection, evaluation, and treatment of high blood pressure: the JNC 7 report. JAMA 289, 2560-2572.

22. Alberti KG, Eckel RH, Grundy SM, et al. (2009) Harmonizing the metabolic syndrome: a joint interim statement of the International Diabetes Federation Task Force on Epidemiology and Prevention; National Heart, Lung, and Blood Institute; American Heart Association; World Heart Federation; International Atherosclerosis Society; and International Association for the Study of Obesity. Circulation 120, 1640-1645.

23. Craig CL, Marshall AL, Sjostrom M, et al. (2003) International physical activity questionnaire: 12 -country reliability and validity. Med Sci Sports Exerc 35, 1381-1395.

24. Xia Y, Xiang Q, Gu Y, et al. (2018) A dietary pattern rich in animal organ, seafood and processed meat products is associated with newly diagnosed hyperuricaemia in Chinese adults: a propensity score-matched case-control study. Br J Nutr 119 , $1177-1184$

25. Wehmeyer MH, Zyriax BC, Jagemann B, et al. (2016) Nonalcoholic fatty liver disease is associated with excessive calorie intake rather than a distinctive dietary pattern. Medicine (Baltimore) 95, e3887. 
26. Hu FB (2002) Dietary pattern analysis: a new direction in nutritional epidemiology. Curr Opin Lipidol 13, 3-9.

27. Linder K, Willmann C, Kantartzis K, et al. (2019) Dietary niacin intake predicts the decrease of liver fat content during a lifestyle intervention. Sci Rep 9, 1303.

28. Chen M, Cheng J, Wu Q, et al. (2018) Prevalence, potential virulence, and genetic diversity of listeria monocytogenes isolates from edible mushrooms in Chinese markets. Front Microbiol 9, 1711.

29. Valverde ME, Hernandez-Perez T \& Paredes-Lopez O (2015) Edible mushrooms: improving human health and promoting quality life. Int J Microbiol 2015, 376387.

30. Jung UJ \& Choi MS (2014) Obesity and its metabolic complications: the role of adipokines and the relationship between obesity, inflammation, insulin resistance, dyslipidemia and nonalcoholic fatty liver disease. Int J Mol Sci 15, 6184-6223.

31. Hsu CH, Liao YL, Lin SC, et al. (2007) The mushroom Agaricus blazei Murill in combination with metformin and gliclazide improves insulin resistance in type 2 diabetes: a randomized, double-blinded, and placebo-controlled clinical trial. J Altern Complement Med 13, 97-102.

32. Martel J, Ojcius DM, Chang CJ, et al. (2017) Anti-obesogenic and antidiabetic effects of plants and mushrooms. Nat Rev Endocrinol 13, 149-160.

33. Zhang S, Tomata Y, Sugiyama K, et al. (2017) Mushroom consumption and incident dementia in elderly Japanese: the Ohsaki Cohort 2006 Study. J Am Geriatr Soc 65 , 1462-1469.

34. Brunt EM, Wong VW, Nobili V, et al. (2015) Nonalcoholic fatty liver disease. Nat Rev Dis Primers 1, 15080.

35. Kim D, Touros A, Kim WR (2018) Nonalcoholic fatty liver disease and metabolic syndrome. Clin Liver Dis 22, 133-140.

36. Agoritsas T, Merglen A, Shah ND, et al. (2017) Adjusted analyses in studies addressing therapy and harm: users' guides to the medical literature. JAMA 317, 748-759. 